\title{
CHARACTERISTIC CLASSES \\ OF TRANSVERSELY HOMOGENEOUS FOLIATIONS
}

BY

CHAL BENSON ${ }^{1}$ AND DAVID B. ELLIS

\begin{abstract}
The foliations studied in this paper have transverse geometry modeled on a homogeneous space $G / H$ with transition functions given by the left action of $G$. It is shown that the characteristic classes for such a foliation are determined by invariants of a certain flat bundle. This is used to prove that when $G$ is semisimple, the characteristic classes are rigid under smooth deformations, extending work of Brooks, Goldman and Heitsch.
\end{abstract}

1. Introduction. Let $\mathscr{F}$ be a codimension- $q$ foliation on a manifold $M$. Then $\mathscr{F}$ can be described by a Haefliger cocycle of submersions from open subsets of $M$ into some model manifold $N$ of dimension $q$. Transverse geometric structures for $\mathscr{F}$ are obtained by requiring that the transition functions for the cocycle preserve a geometric structure on $N$. We consider the case of transversely homogeneous (or $(G, G / H)$ )-foliations; that is where $G$ is a Lie group and $H$ is a closed subgroup, $N=G / H$ and the transition functions are given by the left action of $G$ on $G / H$.

In this paper we examine the characteristic classes of $(G, G / H)$-foliations by exploiting special properties of the normal bundle $\nu \mathscr{F}$ of such a foliation. This is done by applying the techniques of Kamber and Tondeur $[10,11]$ to show that the characteristic classes are determined by invariants of certain bundles associated to $\nu \mathscr{F}$.

Our main application is the following

1.1 Rigidity TheOREM. Let $G$ be a semisimple Lie group. Then the characteristic classes for $(G, G / H)$-foliations are rigid under smooth deformations. That is if $\left\{\mathscr{F}_{t}\right\}$ is a smooth family of $(G, G / H)$-foliations on $M$, then the characteristic classes of $\mathscr{F}_{t}$ (in $\left.H^{*}(M ; \mathbf{R})\right)$ are independent of $t$.

For a discussion of rigid and variable classes we refer the reader to [6]. For examples of nontrivial variable classes see $[7,16]$.

A stronger version of this theorem is proved by Brooks and Goldman in [3] for the special case of $\left(\operatorname{PSL}(2, \mathbf{R}), S^{1}\right)$-foliations and extended to the case of $\left(\operatorname{PSL}(q+1, \mathbf{R}), S^{q}\right)$-foliations by Heitsch (see [8]). This special case is of interest since there are examples of nontrivial classes in this setting. Another special case of

Received by the editors August 23, 1984.

1980 Mathematics Subject Classification. Primary 57R32: Secondary 53C30.

' Partially supported by a UMSL research grant. 
interest is the transversely conformal case, that is $\left(O(q+1,1), S^{q}\right)$-foliations. For examples of nontrivial characteristic classes in both of these cases see [13].

The content of this paper can be summarized as follows. $\$ 2$ contains the definition and some examples of $(G, G / H)$-foliations along with the construction of certain bundles $P \subset P_{G}$ associated to a $(G, G / H)$-foliation. $\S 3$ is a brief review of the Kamber-Tondeur construction of characteristic classes for foliations and flat bundles. In $\$ 4$ the main technical lemma (Lemma 4.1) is proved and used to study framed $(G, G H)$-foliations. This lemma is then applied in $\S 5$ to relate the characteristic classes to the invariants of the pair $\left(P_{G}, P\right)$. The rigidity theorem is proved in $\S 6$.

In this paper all manifolds and foliations are $C^{\infty}$ and all cohomology groups have real coefficients. When $M$ is a manifold, $H^{*}(M)$ should be thought of as the (deRham) cohomology of the complex $(\Omega(M), d)$ of smooth forms on $M$. Throughout $G$ will denote a Lie group, $H \subset G$ a closed subgroup and $\mathfrak{g}$ and $\mathfrak{h}$ will denote the corresponding Lie algebras. We set $q=\operatorname{dim}(G / H)$; thus a $(G, G / H)$-foliation has codimension $q$.

We would like to express our gratitude to R. Blumenthal, S. Hurder, F. Kamber and $\mathrm{P}$. Tondeur for helpful discussions during the preparation of this paper.

2. Properties of transversely homogeneous foliations. We begin this section with the definition of a transversely homogeneous or $(G, G / H)$-foliation and two important examples. Example 2.2 is simply the foliation of $G$ by cosets of $H$, while Example 2.3 is that of a foliation transverse to the fibers of a flat $G$-bundle with fibre $G / H$. The second half of this section is devoted to giving two alternative descriptions of $(G, G / H)$-foliations which can be used to relate the characteristic classes of a general $(G, G / H)$-foliation to the characteristic classes of foliations as in Examples 2.2 and 2.3. The first of these is used in both [ 3 and 8 ] to obtain results on $\left(\operatorname{PSL}(q+1), S^{q}\right)$-foliations; the second will be used in this paper to study the general case.

2.1 Definition. A $(G, G / H)$-foliation $\mathscr{F}$ on $M$ is given by a collection (Haefliger cocycle) $\left(\left\{U_{\alpha}\right\},\left\{f_{\alpha}\right\},\left\{g_{\alpha \beta}\right\}\right)$, where $\left\{U_{\alpha}\right\}$ is an open cover of $M, f_{\alpha}: U_{\alpha} \rightarrow G / H$ is a submersion defining $\mathscr{F} \mid U_{\alpha}$, and $g_{\alpha \beta} \in G$ with $f_{\beta}=g_{\beta \alpha} f_{\alpha}$ on $U_{\alpha} \cap U_{\beta}$.

As examples we have

2.2 Example. The foliation $\mathscr{F}(G, H)$ of $G$ by $H$-cosets $g H$ is a $(G, G / H)$-foliation. More generally, if $\Gamma \subset G$ is discrete, then $\Gamma / G$ carries a $(G, G / H)$-foliation $\mathscr{F}(\Gamma, G, H)$ by left $H$-cosets. The foliation $\mathscr{F}(G, H)$ is defined by the usual submersion $\pi: G \rightarrow G / H$ and its principal normal bundle $\operatorname{GL}(\nu \mathscr{F}(G, H))$ is given by

$$
\operatorname{GL}(\nu \mathscr{F}(G, H))=\pi^{*}(\mathrm{GL}(G / H))=\pi^{*}(G) \times{ }_{H} \mathrm{GL}(\mathfrak{g} / \mathfrak{h}) .
$$

Here $H$ acts on $\operatorname{GL}(\mathfrak{g} / \mathfrak{h})$ by the isotropy representation $\lambda: H \rightarrow \operatorname{GL}(\mathfrak{g} / \mathfrak{h})$. Since $\pi^{*}(G)$ is canonically trivial, $\nu \mathscr{F}(G, H)$ has a standard framing which we denote by

$$
s_{\imath}: G \rightarrow \operatorname{GL}(\nu \mathscr{F}(G, H)) \text {. }
$$

This framing is left invariant so that $\mathscr{F}(\Gamma, G, H)$ is also canonically framed. 
2.3 Example. A homomorphism $\varphi: \pi_{1}(B) \rightarrow G$ can be used to construct a flat bundle

$$
M=\tilde{B} \times_{\varphi}(G / H) \rightarrow B,
$$

where $\tilde{B}$ is the universal cover of $B$. Now $M$ admits a standard "flat" foliation which comes from the product foliation on $\tilde{B} \times G / H$. This is a $(G, G / H)$-foliation since $M$ is locally a product $U \times G / H$ and the transition functions are given by $\varphi$ which acts by left multiplication on $G / H$.

We now indicate how any transversely homogeneous foliation can be related to the foliations in Examples 2.2 and 2.3. Let $\mathscr{F}$ be a $(G, G / H)$-foliation on $M$ given by a cocycle $\left(\left\{U_{\alpha}\right\}\left\{f_{\alpha}\right\},\left\{g_{\alpha \beta}\right\}\right)$ as in Definition 2.1. Then the principal normal bundle $\mathrm{GL}(\nu \mathscr{F})$ of $\mathscr{F}$ is given locally by

$$
\mathrm{GL}(\nu \mathscr{F}) \mid U_{\alpha} \cong f_{\alpha}^{*}(\mathrm{GL}(G / H)) \cong f_{\alpha}^{*}(G) \times{ }_{H} \mathrm{GL}(\mathrm{g} / \mathfrak{h}),
$$

where $H$ acts by isotropy on $\operatorname{GL}(\mathfrak{g} / \mathfrak{h})$ as in Example 2.2. Notice that the transition functions of the cocycle, which are all given by left multiplication, preserve the $H$-reduction $G \rightarrow G / H$ of the frame bundle $\operatorname{GL}(G / H)$. Thus $\operatorname{GL}(\nu \mathscr{F})$ has a canonical $H$-reduction $P \subset \mathrm{GL}(\nu \mathscr{F})$ given locally by

$$
P \mid U_{\alpha} \cong f_{\alpha}^{*}(G) \text {. }
$$

The principal $H$-bundle $P \rightarrow M$ is said to be a foliated reduction of the principal normal bundle $\operatorname{GL}(\nu \mathscr{F})$ since it arises from an $H$-structure on the model manifold which is preserved by a cocycle. In [11] it is shown that this idea can be expressed in various ways; in particular, a foliated reduction is one that admits a Bott connection - that is, a connection that induces a Bott connection in $\operatorname{GL}(\nu \mathscr{F})$.

It is natural to consider the $G$-prolongation of $P$ :

$$
P_{G}=P \times{ }_{H} G \text {. }
$$

This can be described easily in terms of the original cocycle as

$$
P_{G}=\left(\coprod_{\alpha} U_{\alpha} \times G\right) / \sim
$$

where $(x, g) \in U_{\alpha} \times G$ is identified with $\left(x, g_{\beta \alpha} g\right) \in U_{\beta} \times G$ for $x \in U_{\alpha} \cap U_{\beta}$. The bundle $P_{G}$ is flat since the horizontal foliations on each $U_{\alpha} \times G$ piece together to yield a foliation on $P_{G}$ transverse to the fibres.

The $H$-reduction $P \subset P_{G}$ can be viewed as a section $\sigma: M=P / H \rightarrow P_{G} / H$. The bundle $P_{G} / H$ is flat with fibre $G / H$ and one can check that the flat foliation pulls back to $\mathscr{F}$ under $\sigma$. This shows that every $(G, G / H)$-foliation is the pull-back of a foliation as in Example 2.2. This idea is used in [ $\mathbf{3}$ and $\mathbf{8}$ ] to study the characteristic classes of $\left(\operatorname{PSL}(q+1, \mathbf{R}), \mathbf{R} P^{q}\right)$-foliations.

In summary we can view a $(G, G / H)$-foliation as being determined by a flat $G$-bundle $E$ with fibre $G / H$ together with a section $s: M \rightarrow E$ transverse to the fibres, or by a foliated $H$-reduction $P$ of $\operatorname{GL}(\nu \mathscr{F})$. These two points of view are seen to be equivalent by noting that $P$ can also be thought of as an $H$-reduction of the principal $G$ bundle $P_{G}$ associated to $E$. It is this observation which allows us to apply the general machinery of Kamber and Tondeur [10] to study the characteristic classes of $\mathscr{F}$. 
We conclude this section with another description of the pair $P \subset P_{G}$. We will use this description to prove the main technical lemma of §4. In [2], Blumenthal constructs a regular covering $\hat{M} \rightarrow M$ for which the lifted foliation $\hat{\mathscr{F}}$ on $\hat{M}$ is defined by a submersion into $G / H$. Moreover, he shows that the automorphisms of $\hat{M}$ are given by a certain discrete subgroup $\Gamma \subset G$ so that there is a homomorphism $\varphi: \pi_{1}(M) \rightarrow \Gamma \subset G$. Lifting $\mathscr{F}$ to a foliation $\tilde{\mathscr{F}}$ on the universal cover $\tilde{M}$, one obtains a submersion

$$
f: \tilde{M} \rightarrow G / H
$$

defining $\tilde{\mathscr{F}}$. This map $f$ turns out to be $\pi_{1}(M)$-equivariant with respect to $\varphi$. The details of this construction, given in [2], will not be needed here.

In terms of this notation, $f^{*}(G)$ is the foliated $H$-reduction of

$$
\operatorname{GL}(\nu \tilde{\mathscr{F}})=f^{*}(G) \times{ }_{H} \operatorname{GL}(\mathrm{g} / \mathfrak{h}),
$$

and the inclusion of $P$ in $\operatorname{GL}(\nu \mathscr{F})$ is given by:

$$
\begin{array}{ccc}
P & \subset & \mathrm{GL}(\nu \mathscr{F}) \\
\| & & \| \\
\pi_{1}(M) \backslash \mathcal{f}^{*}(G) & \subset & \pi_{1}(M) \backslash \mathrm{GL}(\nu \tilde{\mathscr{F}})
\end{array}
$$

The bundle $P_{G}$ can now be written as

$$
\begin{aligned}
P_{G} & =\pi_{1}(M) \backslash f^{*}(G) \times{ }_{H} G \\
& \cong f^{*}(G) / H \times \times_{\pi_{1}(M)} G=\tilde{M} \times{ }_{\varphi} G
\end{aligned}
$$

which is visibly flat.

3. Background on characteristic classes. The literature contains several equivalent constructions for the characteristic classes of a foliation. For our purposes, the theory developed by Kamber and Tondeur is the most useful since it also produces invariants for foliated reductions and flat bundles. In this section, we will briefly outline their construction to fix some notation. Details can be found in [10 or 11].

Let $\mathscr{F}$ be a codimension- $q$ foliation on $M$ with principal normal bundle GL( $\nu \mathscr{F})$. Using any Bott connection $\beta$ in $\operatorname{GL}(\nu \mathscr{F})$, one obtains the Weil homomorphism

$$
\Delta(\beta): W(\mathrm{gl}(q))_{q} \rightarrow \Omega(\mathrm{GL}(\nu \mathscr{F})),
$$

where $W(\mathfrak{g l}(q))_{q}$ is the quotient of the Weil algebra $\Lambda\left(\mathfrak{g l}(q)^{*}\right) \otimes S\left(\mathfrak{g l}(q)^{*}\right)$ by the ideal generated by symmetric elements of degree $>q$. We will also write $W_{q}$ for this algebra, although in the literature this usually denotes a certain cohomologically equivalent subalgebra.

If $\nu \mathscr{F}$ has a framing $s: M \rightarrow \operatorname{GL}(\nu \mathscr{F})$, then one obtains a well-defined characteristic map

$$
\Delta(\mathscr{F}, s): H^{*}\left(W_{q}\right) \rightarrow H^{*}(M)
$$

given by $s^{*} \circ \Delta(\beta)$ on the form level. In general, since $\Delta(\beta)$ preserves certain actions of $\operatorname{GL}(q)$ and $\mathfrak{g l}(q)$ on $W_{q}$ and $\Omega(\mathrm{GL}(\nu \mathscr{F})$ ) (see [10]), we obtain a characteristic map

$$
\Delta(\mathscr{F}): H^{*}\left(W O_{q}\right) \rightarrow H^{*}(M),
$$


where $W O_{q} \subset W_{q}$ is the subcomplex of $O(q)$-basic elements (those fixed by the action of $O(q)$ and annihilated by the action of its Lie algebra).

If $P \subset \mathrm{GL}(\nu \mathscr{F})$ is a foliated $H$-reduction and $s: M \rightarrow P$ is a section (an $H$-framing for $\nu \mathscr{F})$, then one obtains a characteristic map

$$
\Delta(\mathscr{F}, s): H^{*}\left(W(\mathfrak{h})_{q}\right) \rightarrow H^{*}(M)
$$

by using a Bott connection in $P$. In general, one has

$$
\Delta(\mathscr{F}): H^{*}\left(W\left(\mathfrak{h}, K_{H}\right)_{q}\right) \rightarrow H^{*}(M),
$$

where $K_{H} \subset H$ is any maximal compact subgroup and $W\left(\mathfrak{h}, K_{H}\right)_{q}$ denotes the $K_{,}$-basic elements in $W(\mathfrak{h})_{q}$.

We will also be considering characteristic classes for flat principal $G$-bundles, $P_{G}=\tilde{M} \times{ }_{\varphi} G$. Given a left invariant form on $G$, one obtains a form on $P_{G}$ in the obvious way. This yields a $G-D G$-algebra map $\Delta_{b}: \Lambda g^{*} \rightarrow \Omega\left(P_{G}\right)$. Given a trivialization $s: M \rightarrow P_{G}$, one obtains

$$
\Delta\left(P_{G}, s\right): H^{*}(\mathrm{~g}) \rightarrow H^{*}(M)
$$

from $s^{*} \circ \Delta_{b}$ on the form level. In general, if $P \subset P_{G}$ is an $H$-reduction, then the composition on $H$-basic elements

$$
\left(\Lambda \mathrm{g}^{*}\right)_{H} \rightarrow \Omega\left(P_{G}\right)_{H} \rightarrow \Omega(P)_{H}=\Omega(P / H)=\Omega(M)
$$

yields a homomorphism

$$
\Delta\left(P_{G}, P\right): H^{*}(\mathfrak{g}, H) \rightarrow H^{*}(M) .
$$

Since $P / K_{H} \rightarrow M$ has contractible fibre $H / K_{H}$, there is also a related homomorphism

$$
\Delta\left(P_{G}, P\right): H^{*}\left(\mathfrak{g}, K_{H}\right) \rightarrow H^{*}(M) .
$$

4. Characteristic classes of framed $(G, G / H)$-foliations. We begin this section with the main technical lemma which will be used in $\$ 5$ to study the characteristic map for a $(G, G / H)$-foliation. We then apply this lemma directly to the case of framed $(G, G / H)$-foliations to obtain a diagram which relates the framed classes of such a foliation $\mathscr{F}$ to the flat classes of the flat bundle $P_{G}$ associated to $\mathscr{F}$.

4.1 Lemma. There exists a mapping $\psi: W(\mathfrak{h})_{q} \rightarrow \Lambda \mathfrak{g} *$ of differential graded algebras such that if $\mathscr{F}$ is $a(G, G / H)$-foliation on $M$ then the diagram

$$
\begin{array}{ccccc}
W_{q} & \stackrel{W(\lambda)}{\rightarrow} & W(\mathfrak{h})_{q} & \stackrel{\psi}{\rightarrow} & \Lambda g^{*} \\
\downarrow \Delta(\bar{\beta}) & & \downarrow \Delta(\beta) & & \downarrow \Delta_{h} \\
\Omega(\mathrm{GL}(\nu \mathscr{F})) & \rightarrow & \Omega(P) & \leftarrow & \Omega\left(P_{G}\right)
\end{array}
$$

is commutative upon passage to cohomology. Here the foliated $H$-reduction $P$ of the flat bundle $P_{G}$ associated to $\mathscr{F}$ are as in (2.1) and (2.2) respectively. The map $W(\lambda)$ is obtained from the isotropy representation $\lambda: H \rightarrow \mathrm{GL}(\mathfrak{g} / \mathfrak{h})$, and $\beta$ is a Bott connection in $P$ with induced connection $\bar{\beta}$ in $\operatorname{GL}(\nu \mathscr{F})$. 
Proof. Recall that $P$ is also an $H$-reduction of $\operatorname{GL}(\nu \mathscr{F})$. Thus the left-hand square in the diagram is commutative as it stands by functoriality of induced connections. We need only consider the right-hand portion of the diagram.

We now define the mapping $\psi$; this is done in such a way that the composition

$$
W_{q} \stackrel{W(\lambda)}{\rightarrow} W(\mathfrak{h})_{q} \stackrel{\psi}{\rightarrow} \Lambda \mathfrak{g}^{*}
$$

gives, in cohomology, the characteristic map for the foliation $\mathscr{F}(G, H)$ of $G$ by cosets of $H$ with its canonical framing. Now the normal frame bundle $\operatorname{GL}(\nu \mathscr{F}(G, H))$ has a foliated $H$-reduction $\pi^{*} G$ (see $\S 2$ ) which is canonically trivial. We identify $T\left(\pi^{*} G\right)=G \times \mathfrak{g} \times H \times \mathfrak{h}$ and let $\alpha: \mathfrak{g} \rightarrow \mathfrak{h}$ be a linear splitting of the exact sequence $0 \rightarrow \mathfrak{h} \rightarrow \mathfrak{g} \rightarrow \mathfrak{g} / \mathfrak{h} \rightarrow 0$. Then $\alpha$ determines a left invariant Bott connection $\omega^{\alpha}$ in $\pi^{*} G$ by

$$
\omega^{\alpha}(g, X, h, Y)=\operatorname{Ad}\left(h^{-1}\right)(\alpha(X))+Y .
$$

(For a description of $\omega^{\alpha}$ as a covariant derivative see [15].) The map $\psi$ is then defined to be the composition

$$
\psi: W(\mathfrak{h})_{q} \stackrel{\Delta\left(\omega^{\alpha}\right)}{\rightarrow} T\left(\pi^{*} G\right) \stackrel{s_{c}^{*}}{\rightarrow} \Lambda g^{*},
$$

where $s_{c}: G \rightarrow \pi^{*} G$ is the canonical framing. The image of $\psi$ lands in $\Lambda g^{*}$ since $\omega^{\alpha}$ is left invariant. We remark that the map induced by $\psi$ on cohomology is both independent of the choice of splitting $\alpha$ and yields the characteristic homomorphism (3.3) in this case.

We proceed to show that with this choice of $\psi$ the diagram commutes on cohomology. As shown in $\S 2$, we can write $P=\pi_{1}(M) \backslash f^{*}(G)$, where $f: \tilde{M} \rightarrow G / H$ is a $\pi_{1}(M)$-equivariant submersion defining the lifted foliation $\tilde{\mathscr{F}}$ on $\tilde{M}$. Lifting $\beta$ yields a $\pi_{1}(M)$-invariant Bott connection in the $H$-bundle $f^{*}(G) \rightarrow \tilde{M}$ which we also denote by $\beta$.

Now let $\eta: T G \rightarrow \mathfrak{h}$ be any connection in $G \rightarrow G / H$. Then $f^{*}(\eta)$ is a Bott connection in $f^{*}(G)$. We obtain a diagram

$$
\begin{array}{lcc}
W(\mathfrak{h})_{q} & \stackrel{\Delta(\eta)}{\rightarrow} & \Omega(G) \\
\Delta(\beta) \searrow & & \swarrow \tilde{f}^{*} \\
& \Omega\left(f^{*} G\right) &
\end{array}
$$

which commutes on cohomology. Here $\tilde{f}: f^{*}(G) \rightarrow G$ covers $f: \tilde{M} \rightarrow G / H$. The map $\Delta(\eta)$ gives rise to the characteristic classes for $\mathscr{F}(G, H)$ with its framing $s_{c}$. Indeed, $\pi^{*}(\eta)$ is a Bott connection in $\pi^{*} G$ and in fact $\Delta(\eta)=s_{c}^{*} \circ \Delta\left(\pi^{*}(\eta)\right)$. It follows that the diagram

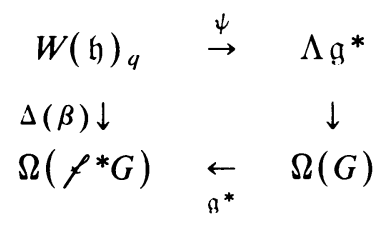

commutes on cohomology. 
To complete the proof note that the bundle $f^{*}(G) \times{ }_{H} G$ is canonically a product $f^{*}(G) / H \times G=\tilde{M} \times G$ and this yields the flat structure. The inclusion $f^{*}(G) \hookrightarrow$ $f^{*}(G) \times{ }_{H} G$ corresponds to the standard inclusion of

$$
f^{*}(G)=\{(x, g) \in \tilde{M} \times G \mid f(x)=g H\}
$$

in $\tilde{M} \times G$. This shows that $\tilde{f}^{*}: \Lambda \mathrm{g}^{*} \rightarrow \Omega\left(f^{*} G\right)$ can also be written as the composition

$$
\Lambda \mathfrak{g}^{*} \underset{\Delta_{b}}{\rightarrow} \Omega\left(f^{*}(G) \times{ }_{H} G\right) \rightarrow \Omega\left(f^{*} G\right) .
$$

Finally, using $\pi_{1}(M)$-invariance of $\beta$ and equivariance of $f$, we can $\bmod$ out by the action of $\pi_{1}(M)$ and conclude that

commutes when we pass to cohomology.

$$
\begin{array}{ccc}
W(\mathfrak{h})_{q} & \stackrel{\psi}{\rightarrow} & \Lambda \mathrm{g}^{*} \\
\Delta(\beta) \downarrow & & \downarrow \Delta_{h} \\
\Omega(P) & \leftarrow & \Omega\left(P_{G}\right)
\end{array}
$$

As an easy application of this lemma, we consider framed $(G, G / H)$-foliations on a manifold $M$. The framing $s$ will be called an $H$-framing if it is really a section in $P \subset \mathrm{GL}(\nu \mathscr{F})$. In this case $s$ is also a section in $P_{G}$ since $P \subset P_{G}$. The following is now obvious from Lemma 4.1 .

4.2 TheOREM. Let $(\mathscr{F}, s)$ be an $H$-framed $(G, G / H)$-foliation on $M$. Then there is a commutative diagram:

$$
\begin{array}{ccc}
H^{*}\left(W_{q}\right) & \stackrel{\Delta\left(\mathscr{F}(G, H), s_{c}\right)}{\rightarrow} & H^{*}(\mathfrak{g}) \\
\Delta(\mathscr{F}, s) \searrow & & \swarrow \Delta\left(P_{G}, s\right) \\
& H^{*}(M) &
\end{array}
$$

The notation $\Delta\left(\mathscr{F}(G, H), s_{c}\right)$ in this theorem is somewhat misleading since this map should land in $H^{*}(G)$. One obtains the characteristic classes for $\left(\mathscr{F}(G, H), s_{c}\right)$ by composing this map with $H^{*}(\mathfrak{g}) \rightarrow H^{*}(G)$. More generally, if $\Gamma \subset G$ is discrete, then $\Delta\left(\mathscr{F}(\Gamma, G, H), s_{c}\right)$ is given by the composition $H^{*}\left(W_{q}\right) \rightarrow H^{*}(\mathrm{~g}) \rightarrow$ $H^{*}(\Gamma \backslash G)$. If $G$ is semisimple, and $\Gamma$ is a cocompact discrete subgroup, then $H^{*}(\mathfrak{g}) \rightarrow H^{*}(\Gamma \backslash G)$ is injective. All of this is of course quite standard. Theorem 4.2 shows that for $G$ semisimple, any class $c \in H^{*}\left(W_{q}\right)$ which is nontrivial for some $H$-framed $(G, G / H)$-foliation must also be nontrivial for some coset foliation $\mathscr{F}(\Gamma, G, H)$. The latter have been studied extensively $[\mathbf{1}, \mathbf{1 5}]$.

It is not hard to find framed $(G, G / H)$-foliations that are not $H$-framed. One can obtain examples by applying the permanence construction [9] to an $H$-framed foliation. Suppose that $\mathscr{F}$ is an $H$-framed $(G, G / H)$-foliation on $M$ and consider the pull-back $\mathscr{F}^{\prime}=p^{*}(\mathscr{F})$ on $M \times \mathrm{GL}(q)$ under the projection $p: M \times \mathrm{GL}(q) \rightarrow M$. This has an $H$-framing $s^{\prime}=p^{*}(s)$ and also a "twisted" framing $s^{\prime \prime}$ defined using the right action of $\operatorname{GL}(q)$ on $\mathrm{GL}\left(\nu \mathscr{F}^{\prime}\right)$,

$$
s^{\prime \prime}(m, A)=s^{\prime}(m) \cdot A \text {. }
$$

The resulting framed $(G, G / H)$-foliation $\left(\mathscr{F}^{\prime}, s^{\prime \prime}\right)$ is not $H$-framed. 
It is shown in [9] that any class $a \in H^{*}\left(W_{q}\right)$ which is nontrivial for $(\mathscr{F}, s)$ gives rise to a whole family of nonvanishing classes for $\left(\mathscr{F}^{\prime}, s^{\prime \prime}\right)$. This indicates that from a characteristic class viewpoint, the framed $(G, G / H)$-foliations are richer than the coset foliations $\mathscr{F}(G, H)$. A natural question to ask is whether every class $a \in$ $H^{*}\left(W_{q}\right)$ which is nontrivial for some framed $(G, G / H)$-foliation can be obtained by permanence from a class which is nontrivial for $\mathscr{F}(G, H)$. Unfortunately we have been unable to give a complete answer to this question. We remark however that for many pairs $(G, H)$ examined in the literature, the nontrivial classes for $\mathscr{F}(G, H)$ are already closed under permanence.

5. Characteristic classes for $(G, G / H)$-foliations. We now use Lemma 4.1 to relate the characteristic classes for a $(G, G / H)$-foliation $\mathscr{F}$ to invariants for its associated flat $G$-bundle with $H$-reduction. These invariants are given by homomorphisms (3.6) and (3.7).

Let $K_{H} \subset H$ be a maximal compact subgroup. We may assume that $\lambda\left(K_{H}\right) \subset O(q)$ (as before, $\lambda: H \rightarrow \mathrm{GL}(q)$ is isotropy) since in any case $\lambda\left(K_{H}\right)$ is contained in some conjugate of $O(q)$. The characteristic homomorphism (3.2), $\Delta(\mathscr{F}): H^{*}\left(W O_{q}\right) \rightarrow$ $H^{*}(M)$, is given by the compostion

$$
H^{*}\left(W O_{q}\right) \stackrel{W(\lambda) *}{\rightarrow} H^{*}\left(W\left(\mathfrak{h}, K_{H}\right)_{q}\right) \stackrel{\Delta(\pi)}{\rightarrow} H^{*}(M)
$$

where the second map is given by (3.4).

To complete the passage to basic elements in the diagram from Lemma 4.1, we must circumvent a technical difficulty. The map $\psi: W(\mathfrak{h})_{q} \rightarrow \Lambda \mathfrak{g}^{*}$, defined as $s_{c}^{*} \circ \Delta\left(\omega^{\alpha}\right)$ for some splitting $\alpha: \mathfrak{g} \rightarrow \mathfrak{h}$, need not preserve the operations of $K_{H}$ and its Lie algebra. However, we have the following lemma.

5.1 Lemma. Let $\alpha: \mathfrak{g} \rightarrow \mathfrak{h}$ be an Ad- $K_{H^{-}}$equivariant splitting of $0 \rightarrow \mathfrak{h} \rightarrow \mathfrak{g} \rightarrow \mathfrak{g} / \mathfrak{h}$ $\rightarrow 0$. Then the map $\psi=s_{c}^{*} \circ \Delta\left(\omega^{\alpha}\right)$, where $\omega^{\alpha}$ is defined by (4.1), preserves the operations of $K_{H}$ and its Lie algebra on $W(\mathfrak{h})_{q}$ and $\Lambda \mathrm{g}^{*}$.

The proof involves tedious but straightfoward computations that will not be presented here.

It is always possible to find a splitting $\alpha$ as in the lemma. Indeed, if $\gamma: \mathfrak{g} \rightarrow \mathfrak{h}$ is any linear splitting, then we obtain an Ad- $K_{H}$-equivariant splitting by averaging over $K_{n}$ with respect to Haar measure,

$$
\alpha(v)=\int_{K_{\prime \prime}} \operatorname{Ad}(k) \gamma\left(\operatorname{Ad}\left(k^{-1}\right) v\right) d k .
$$

These remarks imply the following result.

5.2 TheOREM. Let $\mathscr{F}$ be a $(G, G / H)$-foliation on $M$ with associated flat $G$-bundle $P_{G}$ and $H$-reduction $P$. Then there is a commutative diagram:

$$
\begin{array}{ccc}
H^{*}\left(W O_{q}\right) & \rightarrow & H^{*}\left(\mathfrak{g}, K_{H}\right) \\
\Delta(\mathscr{F}) \searrow & & \swarrow\left(P_{(i}, P\right) \\
& H^{*}(M) &
\end{array}
$$


This shows how the characteristic classes for $\mathscr{F}$ are determined in a natural way by those for its associated flat G-bundle with H-reduction.

One is tempted to pass to $\mathrm{GL}(q)$ and $H$-basic elements and obtain the Pontrjagin classes for the normal bundle $\nu \mathscr{F}$. However, this would require $\alpha$ to be Ad- $H$ equivariant. In general, such a splitting will not exist and one must require that $(G, H)$ be a reductive pair (see [10]).

5.3 TheOREM. Let $\mathscr{F}$ be as in Theorem 5.2, where $(G, H)$ is a reductive pair. Then

$$
\operatorname{Pont}^{*}(\nu \mathscr{F}) \subset \operatorname{Im}\left(H^{*}(\mathfrak{g}, H) \stackrel{\Delta\left(P_{G}, P\right)}{\rightarrow} H^{*}(M)\right) .
$$

It would be interesting to know under what generality this theorem holds. A procedure similar to that given by (4.1) shows that an Ad- $H$-equivariant splitting yields a left $G$-invariant connection in the $H$-bundle $G \rightarrow G / H$. We have been able to prove Theorem 5.3 under the slightly weaker assumption that $\operatorname{GL}(G / H)$ admits a left $G$-invariant connection. Such connections are given by a splitting $\alpha$ satisfying a condition weaker than Ad- $H$-equivariance (see Chapter 10 of [12]).

6. The rigidity theorem. We turn to the proof of Theorem 1.1. The proof uses the notion of the cohomology $H^{*}(\mathfrak{g}, V)$ of a Lie algebra $\mathfrak{g}$ with coefficients in a $G$-module $V$. This is the cohomology of the complex $(\Lambda(\mathrm{g}, V), d)$, where

$$
\Lambda^{k}(\mathrm{~g}, V)=\left\{f: \mathrm{g}^{k} \rightarrow V \mid f \text { is } k \text {-multilinear and alternating }\right\}
$$

and

$$
\begin{aligned}
d f\left(X_{1}, \ldots, X_{k+1}\right)= & \sum_{i<j}(-1)^{i+j} f\left(\left[X_{i}, X_{j}\right], X_{1}, \ldots, \hat{X}_{i}, \ldots, \hat{X}_{j}, \ldots, X_{k+1}\right) \\
& +\sum_{i}(-1)^{i+1} X_{i} \cdot f\left(X_{1}, \ldots, \hat{X}_{i}, \ldots, X_{k+1}\right) .
\end{aligned}
$$

There are actions of $G$ and $g$ on $\Lambda(g, V)$ given by

$$
(g \cdot f)\left(X_{1}, \ldots, X_{k}\right)=g \cdot f\left(\operatorname{Ad}\left(g^{-1}\right) X_{1}, \ldots, \operatorname{Ad}\left(g^{-1}\right) X_{k}\right)
$$

and

$$
(X \cdot f)\left(X_{1}, \ldots, X_{k-1}\right)=f\left(X, X_{1}, \ldots, X_{k-1}\right) .
$$

These actions enable one to define the relative cohomology $H^{*}(\mathrm{~g}, A, V)$ for a closed subgroup $A \subset G$. These algebraic notions are described in [4].

Proof of Theorem 1.1. Let $\left\{\mathscr{F}_{t}\right\}$ be a smooth family of $(G, G / H)$-foliations on $M$. The foliated $H$-reduction of $\operatorname{GL}\left(\nu \mathscr{F}_{t}\right)$ is, say, $P^{t}$ with flat $G$-prolongation $P_{G}^{t}$. Theorem 5.2 shows that $\Delta\left(\mathscr{F}_{t}\right)$ can be written as

$$
H^{*}\left(W O_{q}\right) \rightarrow H^{*}\left(\mathrm{~g}, K_{H}\right) \underset{\Delta\left(P_{G}^{\prime}, P^{\prime}\right)}{\rightarrow} H^{*}(M) .
$$

The $\mathscr{F}_{t}$ 's fit together to form a codimension- $(q+1)$ foliation on $M \times \mathbf{R}$. This produces bundle isomorphisms between all the $P^{t}$ 's and all the $P_{G}^{t}$ 's. We will identify all the $P^{t}$ 's with a single $H$-bundle $P$ and all the $P_{G}^{t}$ 's with a single $G$-bundle $P_{G}$. Of 
course $P_{G}$ carries a 1-parameter family of flat structures. The map $\Delta\left(P_{G}^{t}, P^{t}\right)$ is induced by the composition

$$
F_{t}: \Lambda g^{*} \stackrel{\Delta_{b}^{t}}{\rightarrow} \Omega\left(P_{G}\right) \rightarrow \Omega(P)
$$

taken on $K_{H}$-basic elements.

To complete the proof, we will differentiate the family of maps $\left\{\Delta\left(P_{G}^{t}, P^{t}\right)\right\}$ with respect to $t$ and show that the result is zero. To simplify notation, we consider only the derivative at $t=0$. It will be shown that

$$
\left.\frac{d}{d t}\right|_{t=0} \Delta\left(P_{G}^{t}, P^{t}\right): H^{k}\left(\mathfrak{g}, K_{H}\right) \rightarrow H^{k}(M)
$$

factors through $H^{k-1}\left(\mathfrak{g}, K_{H}, \mathrm{~g}^{*}\right)$, a relative cohomology with coefficients in the $G$-module $\mathrm{g}^{*}$ (under the contragredient Adjoint action). This trick was first used in the early 70's in the work of the "Russian school" of foliation theory. For a detailed analysis of this idea, we refer the reader to [5 or 14].

We define a map Var: $\Lambda^{k}\left(\mathfrak{g}^{*}\right) \rightarrow \Lambda^{k-1}\left(\mathfrak{g}, \mathfrak{g}^{*}\right)$ by

$$
\operatorname{Var}(f)\left(X_{1}, \ldots, X_{k-1}\right)(Y)=f\left(X_{1}, \ldots, X_{k-1}, Y\right) \text {. }
$$

Let $\left\{\omega_{1}, \ldots, \omega_{n}\right\}$ be a basis for $\mathrm{g}^{*}$. Then the elements of $\Lambda^{k-1}\left(\mathfrak{g}, \mathrm{g}^{*}\right)$ can be written as sums of terms $f \otimes \omega_{i}$, where $f \in \Lambda^{k-1}\left(\mathrm{~g}^{*}\right)$. We define a map $\dot{F}: \Lambda^{k-1}\left(\mathrm{~g}, \mathrm{~g}^{*}\right) \rightarrow$ $\Omega^{k}(P)$ by

$$
\dot{F}\left(f \otimes \omega_{i}\right)=\left.F_{0}(f) \wedge \frac{d}{d t}\right|_{t=0} F_{t}\left(\omega_{i}\right) .
$$

Then

$$
\left.\frac{d}{d t}\right|_{t=0} F_{t}(f)=\dot{F}(\operatorname{Var}(f)) .
$$

The maps Var and $\dot{F}$ commute with the differentials. Moreover, Var is compatible with the actions of $G$ and $g$ on $\Lambda g^{*}$ and $\Lambda\left(g, g^{*}\right)$, and $\dot{F}$ is compatible with the actions of $H$ and $\mathfrak{g}$ on $\Lambda\left(\mathfrak{g}, \mathfrak{g}^{*}\right)$ and $\Omega(P)$. This shows that we have a factoring

$$
\left.\frac{d}{d t}\right|_{t=0} \Delta\left(P_{G}^{t}, P^{t}\right): H^{k}\left(\mathrm{~g}, K_{H}\right) \stackrel{\mathrm{Var}_{*}}{\rightarrow} H^{k-1}\left(\mathrm{~g}, K_{H}, \mathrm{~g}^{*}\right) \stackrel{\dot{F}_{*}}{\rightarrow} H^{k}\left(P / K_{H}\right)=H^{k}(M) .
$$

This completes the proof since it is known that when $\mathfrak{g}$ is semisimple, $H^{*}\left(\mathrm{~g}, A, \mathrm{~g}^{*}\right)=0$ for any closed subgroup $A \subset G$. Indeed, it is shown in [4] that $H^{*}(\mathfrak{g}, A, V)=0$ for any nontrivial irreducible $G$-module $V$ and the module $\mathfrak{g}^{*}$ splits into a direct sum of irreducible modules when $\mathfrak{g}$ is semisimple.

We remark that the proof in fact shows rigidity for the characteristic classes of $(G, G / H)$-foliations provided that $(G, H)$ satisfies $H^{*}\left(\mathfrak{g}, K_{H}, \mathfrak{g}^{*}\right)=0$. We should note however that this proof fails to generalize the full strength of the Brooks-Goldman and Heitsch results (for $\left(\operatorname{PSL}(q+1, \mathbf{R}), S^{q}\right)$-foliations) since they show that there are only finitely many possible values for the characteristic classes in these cases. It is natural to conjecture that this is true in general (for $G$ semisimple at least) but we have been unable to prove this. 


\section{REFERENCES}

1. D. Baker, On a class of foliations and the evaluation of their characteristic classes, Comment. Math. Helv. 53 (1978), 334-363.

2. R. Blumenthal, Transversely homogeneous foliations, Ann. Inst. Fourier (Grenoble) 29 (1979), $143-158$.

3. R. Brooks and W. Goldman, The Godbillon-Vey invariant of a transversely homogeneous foliation, Trans. Amer. Math. Soc. 286 (1984), 651-664.

4. C. Chevalley and S. Eilenberg, Cohomology theory of Lie groups and Lie algebras, Trans. Amer. Math. Soc. 63 (1948), 85-124.

5. D. B. Fuks, Cohomology of infinite dimensional Lie algebras and characteristic classes for foliations, J. Soviet Math. 11 (1979), 922-980.

6. J. Heitsch, Deformations of secondary characteristic classes, Topology 12 (1973), 381-388.

7. __ Independent variation of secondary classes, Ann. of Math. (2) 108 (1978), 421-460.

8 . Secondary invariants of transversely homogeneous foliations, preprint.

9. S. Hurder, On the secondary classes of foliations with trivial normal bundles, Comment. Math. Helv. 56 (1981), 307-326.

10. F. W. Kamber and Ph. Tondeur, Foliated bundles and characteristic classes, Lecture Notes in Math., vol. 493, Springer-Verlag, Berlin and New York, 1975.

11. __ G-foliations and their characteristic classes, Bull. Amer. Math. Soc. 84 (1978), 1086-1124.

12. S. Kolayashi and K. Nomizu, Foundations of differential geometry, Vol. 2, Wiley, New York, 1969.

13. S. Nishikawa and H. Sato, On characteristic classes of Riemannian, conformal and projective foliations, J. Math. Soc. Japan 28 (1976), 224-241.

14. H. Pittie, Characteristic classes of foliations, Pitman, London, 1976.

15. The secondary characteristic classes of parabolic foliations, Comment. Math. Helv. 54 (1979), $601-614$

16. W. P. Thurston, Non cobordant foliations of $S^{3}$, Bull. Amer. Math. Soc. 78 (1972), 511-514.

Department of Mathematical Sciences, University of Missouri, St. Louis, Missouri 63121 (Current address of Chal Benson)

Department of Mathematics, Washington University, St. Louis, Missouri 63130

Current address (D. B. Ellis): Department of Mathematics, Stetson University, Deland, Florida 32720 